\title{
Special issue on cloud computing for scientific and business needs
}

\author{
Arun Agarwal $^{1} \cdot$ Rajkumar Buyya $^{2,3}$
}

Published online: 15 November 2017

(c) CSI Publications 2017

Cloud computing is service oriented utility computing model to deliver computing, platform, and applications as services to the end users based on pay and use consumption model. Cloud technologies are no more hype, and the vision of Cloud computing has come to reality by having the delivery of Cloud services across the globe.

As Big Data demand large scale computing and storage infrastructures for processing and data exploitations, Cloud infrastructures will be next generation back-end computing paradigm for the development of analytics for both scientific and business computing. International Data Corporation (IDC) report predicted that there could be an increase of digital data by 40 times from 2012 to 2020 .

This special issue is devoted to addressing topics in Cloud Computing for both business and scientific computing areas. We intended to bring together researchers, practitioners, developers, and users who are interested in Cloud computing to explore new ideas, techniques, and tools, as well as to exchange their experiences.

This issue received 13 submissions and 6 accepted papers are noted below:

Arun Agarwal

aruncs@uohyd.ernet.in

Rajkumar Buyya

rbuyya@unimelb.edu.au

1 School of Computer and Information Sciences, University of Hyderabad, Hyderabad 500046, India

2 Cloud Computing and Distributed Systems Lab, The University of Melbourne, Melbourne, Australia

3 Manjrasoft Pvt. Ltd., Melbourne, Australia
1. Dynamic algorithm modeling application for comparing distributed computing frameworks

2. Unified resource management in cloud based data centers

3. Application-aware network: network route management using SDN based on application characteristics

4. Cloud resource reduction evaluation by video caching and streaming in LAN environment

5. Split key management framework for OpenStack swift object storage cloud

6. A curious collaborative approach for data integrity verification in cloud computing

The first two papers are invited papers and the remaining are regular papers, which are accepted after peer review.

We thank Prof. S. V. Raghavan and the technical team of CSI ICT for their support. We also thank all the authors for their submission and peer reviewers for their support in selection of the above six papers. 